\title{
An inverse approach for ultrasonic imaging from full matrix capture data. Application to resolution enhancement in NDT.
}

\author{
Nans Laroche, Sébastien Bourguignon, Ewen Carcreff, Member, IEEE, Jérôme Idier, Member, IEEE, \\ and Aroune Duclos
}

\begin{abstract}
In the context of nondestructive testing (NDT), this paper proposes an inverse problem approach for the reconstruction of high-resolution ultrasonic images from full matrix capture (FMC) datasets. We build a linear model that links the FMC data, i.e. the signals collected from all transmitter-receiver pairs of an ultrasonic array, to the discretized reflectivity map of the inspected object. In particular, this model includes the ultrasonic waveform corresponding to the transducers response. Despite the large amount of data, the inversion problem is illposed. Therefore, a regularization strategy is proposed, where the reconstructed image is defined as the minimizer of a penalized least-squares cost function. A mixed penalization function is considered, which simultaneously enhances the sparsity of the image (in NDT, the reflectivity map is mostly zero except at the flaw locations) and its spatial smoothness (flaws may have some spatial extension). The proposed method is shown to outperform two well-known imaging methods: the Total Focusing Method (TFM) and Excitelet. Numerical simulations with two close reflectors show that the proposed method improves the resolution limit defined by the Rayleigh criterion by a factor of four. Such high-resolution imaging capability is confirmed by experimental results obtained with side drilled holes in an aluminum plate.
\end{abstract}

\section{INTRODUCTION}

$\mathbf{U}$ LTRASONIC imaging is widely used in non destructive testing (NDT) [1], medical imaging [2] and structural health monitoring (SHM) [3]. From decades, array probes have been extensively used, due to their ability to form images [4] and to characterize flaws [5]. The conventional way to perform ultrasonic imaging with arrays is hardware (HW) beamforming, which consists in applying specific time delays to each element, in order to focus at particular locations of the specimen under test [1]. Such HW methods have lower performance in terms of resolution and signal-to-noise ratio than software (SW) beamforming techniques, which perform the beamforming in post processing by delaying and averaging unfocused signals at each pixel of the image [6]. Due to the increasing performance of ultrasonic hardware and Graphics Processing Units (GPU), SW beamforming techniques have become a standard for real-time imaging in industrial NDT [7] and medical imaging [8].

N. Laroche and E. Carcreff are with the Phased Array Company (TPAC), Nantes, France (e-mail: nans.laroche@tpac-ndt.com).

$\mathrm{N}$. Laroche, S. Bourguignon, and J. Idier are with the Laboratoire des Sciences du Numérique de Nantes (LS2N), Nantes, France.

A. Duclos is with the Laboratoire d'Acoustique de l'Universite du Mans (LAUM), Le Mans, France.
The Total Focusing Method (TFM) [6], [9] considers Full Matrix Capture (FMC) data, which is the set of signals collected by all transmitter-receiver pairs. It is a standard delay and suam (DAS) reconstruction technique operating linearly on time-domain signals. Several variants of DAS methods have been proposed, which differ by their acquisition process, such as the Synthetic Aperture Focusing Technique (SAFT) [10], Plane Wave Imaging (PWI) [11] or Virtual Source Aperture (VSA) [12], [13]. Frequency-domain variants of these methods have been developed in seismology [14], and were applied to medical imaging [15] and NDT [16], [17]. Although they rely on different data acquisition schemes, their beamforming is based on the same principle, which consists in summing the collected signals at the proper times of flight.

Ultrasonic transducers pulse and receive ultrasonic signals in a limited bandwidth. Thus, the time-domain response of a scatterer has an oscillatory nature and is temporally spread. Images produced by DAS methods, which simply sum the delayed signals, therefore suffer from spatial spreading, and their resolution is limited by the Rayleigh criterion, which defines the acoustical and geometrical resolution limits of an imaging system [18], [19]. Considering the shape of such oscillating waveform in the reconstruction method may then be an efficient lever to improve the quality of reconstructed images. The Excitelet algorithm [20], for example, uses the correlation between the measured signals and the impulse response of the transducers, and can be interpreted as a matched filtering procedure, which increases the contrast in the image. However, the image produced by such a linear method still contains oscillations, and therefore remains limited in resolution.

In order to increase the resolution, non-linear methods are required. In particular, regularization methods aim to compensate the loss of high-frequency information caused by the narrow bandwidth of the transducers, by incorporating specific prior information. For example, sparsity has been used in ultrasonic imaging in the context of SAFT reconstruction [21], [22]. In medical imaging, inverse problems have been formulated for standard beamforming [23] and PWI [24]. Nevertheless, none of these methods integrates the acoustic waveform in its model. This waveform, that will be called the elementary signature in this paper, can be defined as the response, in the time-domain signal, of a scatterer in the material. An ultrasonic signal is then modeled as the convolution of this elementary signature and the reflectivity 
function of the material under test [25], [26].

In this paper, this model is extended to each signal of FMC data by building an appropriate waveform matrix that includes the elementary signature. Thus, the FMC data is linked to the spatial distribution of the acoustic reflectivity inside the inspected medium. An inverse problem is then formulated, and regularization is performed by imposing both the sparsity and the spatial smoothness of the image. Similar methods have been developed in the context of PWI data for medical imaging applications [27], [28]. Here, the proposed inverse method is applied to FMC data for the separation of close scatterers in NDT. Despite their large size, FMC data are more commonly used in the context of NDT applications. Moreover, the resolution of TFM images is naturally better than PWI images [29] and TFM is hence more adapted to the difficult challenge of separating close flaws. The separation of closely spaced flaws is a crucial issue for several NDT applications, such as the separation of close porosities or the detection of small cracks close to the surface of the piece under inspection. Indeed, the lack of resolution of imaging methods becomes critical when the data contain overlapping echoes created by close scatterers. For a low level of uncorrelated noise, a super resolution technique based on the Time Reversal with Multiple Signal Classification (TR-MUSIC) was shown to achieve better results than standard techniques in NDT [19], [30]-[32] and in medical imaging [33]. Nevertheless, this method is highly affected by uncorrelated noise [19] and is restricted to the detection of a known number of point-like scatterers.

This paper is organized as follows. Section II introduces the TFM and Excitelet linear reconstruction methods, and discusses their limitations. The proposed data model is detailed in Section III, and a dedicated inversion procedure is developed in Section IV. In Section V, our approach is compared to standard linear methods on synthetic data composed of overlapping echoes. Section VI evaluates the method on experimental FMC data obtained from a material containing close flaws to be detected. A discussion is finally given in Section VII.

\section{LINEAR ULTRASONIC IMAGING METHODS}

Full Matrix Capture (FMC) consists in recording the signals from all emitter-receiver pairs of transducers in a phased array. Therefore, for an array of $N_{\mathrm{el}}$ transducers, $N_{\mathrm{el}}^{2}$ signals are received. Let $y_{i, j}(t)$ denote the A-scan signal corresponding to the $i$-th transmitter and the $j$-th receiver. Figure 1 shows the path of the ultrasonic wave from transmitter $i$ (with coordinates $\left(u_{i}, 0\right)$ ) to receiver $j$ (with coordinates $\left(v_{j}, 0\right)$ ), through a potential scatterer located at coordinates $(x, z)$.

The TFM is a standard method in NDT in order to process FMC data. The focusing is performed at each point $(x, z)$ of the image by summing all signals at the corresponding times of flight $\tau(i, j, x, z)$. The reconstructed image then reads:

$$
O_{\mathrm{TFM}}(x, z)=\sum_{i=1}^{N_{\mathrm{el}}} \sum_{j=1}^{N_{\mathrm{el}}} y_{i, j}(\tau(i, j, x, z)) .
$$

This operation is computationally expensive, but all pixel intensities can be computed in parallel in order to achieve realtime computation of the whole image with GPU [34]. Times

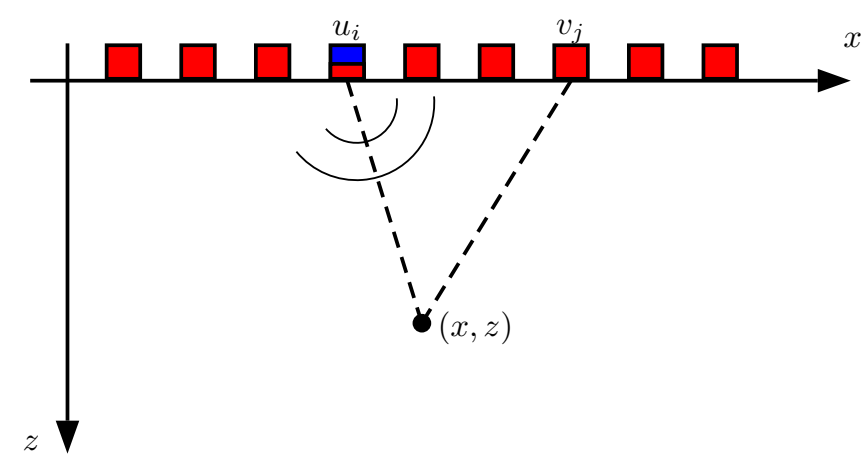

Fig. 1. FMC data acquisition. The signal is emitted by the element $i$ in blue to a potential scatterer located in $(x, z)$, and the reflected signal is received by all elements in red.

of flight are computed by satisfying Fermat's principle [35], and depend on the inspection geometry and on the properties of the inspected material. When the probe is in contact with a flat specimen, they can be obtained straightforwardly by:

$$
\tau(i, j, x, z)=\frac{\sqrt{\left(x-u_{i}\right)^{2}+z^{2}}+\sqrt{\left(x-v_{j}\right)^{2}+z^{2}}}{c},
$$

where $c$ is the sound velocity in the material (which is supposed homogeneous). For a layered isotropic medium, times of flight can be computed using an optimization algorithm [36] or by analytical results for flat surfaces [37]. For anisotropic materials, they can be computed using the Shortest Path Raytracing method [38] or the Fast Marching Method [39], [40].

The Excitelet algorithm [20] is also a post-processing algorithm that focuses at each point of the reconstruction grid. It can be viewed as a matched filtering procedure, which correlates the measured data with the elementary signature in order to improve the detection and localization performance of point scatterers in the data. The intensity at each pixel of the image is computed by:

$$
O_{\mathrm{EXC}}(x, z)=\sum_{i=1}^{N_{\mathrm{el}}} \sum_{j=1}^{N_{\mathrm{el}}}\left(y_{i, j} * h\right)(\tau(i, j, x, z)),
$$

where $*$ denotes the convolution and $h$ is the elementary signature considered in the model. The Excitelet algorithm is usually associated with a thresholding step in order to detect flaws. Nevertheless, $O_{\text {EXC }}$ is a linear function of the data and the thresholding step cannot resolve two flaws that appear as a single spot in the Excitelet image. Therefore, its resolution remains limited.

\section{FORWARD MODEL}

This section aims to describe the acquisition process involved in ultrasonic FMC data [41]. In Subsection III-A, a linear model on a single A-scan using the elementary signature is described. Then, this model is inserted into the description of FMC data by building the corresponding waveform matrix. Then, Subsection III-B discusses the model that will be used for the elementary signature in the waveform matrix. 
$$
\mathbf{H}_{i, j}^{i_{x}}=\underbrace{\left[\begin{array}{cccc}
\boldsymbol{h}_{i, j}^{i_{x}, 1} & \vdots & \ldots & 0 \\
0 & 0 & \ddots & \vdots \\
\vdots & \boldsymbol{h}_{i, j}^{i_{x}, 2} & \ddots & \vdots \\
\vdots & 0 & \ddots & \vdots \\
\vdots & \vdots & \ddots & 0 \\
\vdots & \vdots & \ddots & \boldsymbol{h}_{i, j}^{i_{x}, N_{z}} \\
0 & \vdots & \ldots & 0
\end{array}\right]}_{N_{z}}\} N_{t}
$$

Fig. 2. Scheme of a block of the waveform matrix.

\section{A. Data model and construction of the waveform matrix}

In this section, we build a model that links the data to the reflectivity map of the medium. The following notations refer to discretized objects. The image is computed on a spatial grid $\left(i_{x}, i_{z}\right)$ containing $N_{x}$ columns and $N_{z}$ rows. An ultrasonic signal is modeled as the convolution of the reflectivity of the medium and the elementary signature $\boldsymbol{h}_{i, j}^{i_{x}, i_{z}} \in \mathbb{R}^{N_{h}}$ of a potential scatterer located at $\left(i_{x}, i_{z}\right)$ [25], [26]. The waveform $\boldsymbol{h}_{i, j}^{i_{x}, i_{z}}$ denotes the signature, in the A-scan $\boldsymbol{y}_{i, j}$, of a potential scatterer located at $(x, z)$. Then, the A-scan $\boldsymbol{y}_{i, j}$ is the summation of all shifted elementary signatures, weighted by the pixel intensity of the corresponding reflectivity map:

$$
\boldsymbol{y}_{i, j}=\sum_{i_{x}=1}^{N_{x}} \sum_{i_{z}=1}^{N_{z}} \widetilde{\boldsymbol{h}}_{i, j}^{i_{x}, i_{z}} o^{i_{x}, i_{z}},
$$

where $\widetilde{\boldsymbol{h}}_{i, j}^{i_{x}, i_{z}} \in \mathbb{R}^{N_{t}}$ is the elementary signature $\boldsymbol{h}_{i, j}^{i_{x}, i_{z}}$ padded with zeros and properly time-shifted. More precisely, let the column vector $\boldsymbol{o}^{i_{x}}$ collect the reflectivity values for all pixels in column $i_{x}$. Equation (4) can be written:

$$
\boldsymbol{y}_{i, j}=\sum_{i_{x}=1}^{N_{x}} \mathbf{H}_{i, j}^{i_{x}} \boldsymbol{o}^{i_{x}}
$$

where $\mathbf{H}_{i, j}^{i_{x}}$ is the $N_{t} \times N_{z}$ matrix whose columns are composed of the elementary signature, shifted by the corresponding times of flight. Figure 2 shows the structure of matrix $\mathbf{H}_{i, j}^{i_{x}}$. The number of zeros $\mathcal{K}_{i, j}^{i_{x}, i_{z}}$ before $\boldsymbol{h}_{i, j}^{i_{x}, i_{z}}$ in each column is equal to:

$$
\mathcal{K}_{i, j}^{i_{x}, i_{z}}=\left(\tau(i, j, x, z)-\frac{t_{h}}{2}\right) F_{s}
$$

where $F_{s}$ is the sampling frequency and $t_{h}$ is the duration of the pulse, which is defined for $t \in\left[-\frac{t_{h}}{2}, \frac{t_{h}}{2}\right]$.

Let us remark that the time of flight $\tau$ does not depend linearly on the depth $z$ of the pixel. Therefore, the index shift between two neighboring columns $\mathcal{K}_{i, j}^{i_{x}, i_{z}+1}-\mathcal{K}_{i, j}^{i_{x}, i_{z}}$ is not constant. In addition, the elementary signature may vary

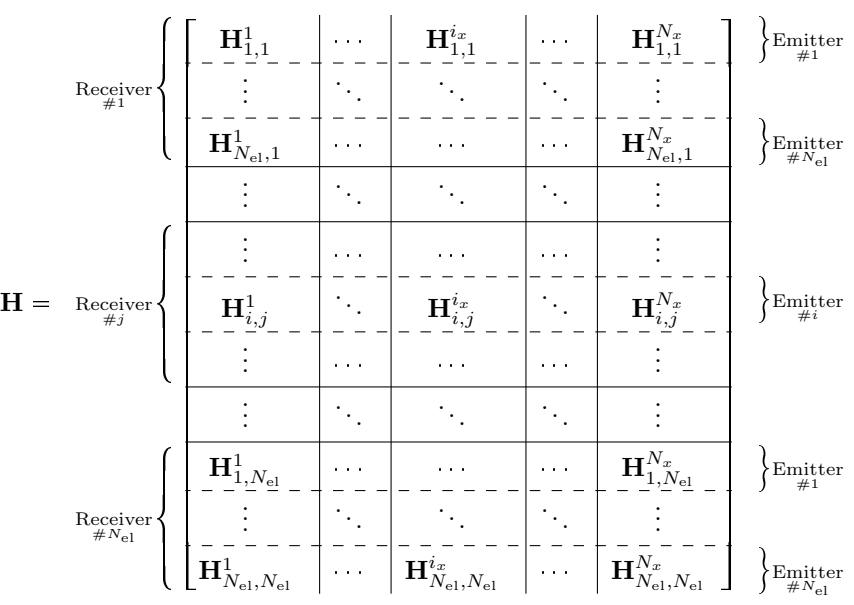

Fig. 3. Scheme of the waveform matrix. Vertical plain lines separate abscissa of the pixel of the reconstruction grid. Horizontal plain lines separate the receiver multiple blocks that each contains $N_{\mathrm{el}}$ blocks of transmitter.

among the columns of $\mathbf{H}_{i, j}^{i_{x}}$ (due for example to attenuation and transducers directivity). Consequently, $\mathbf{H}_{i, j}^{i_{x}}$ is not a convolution matrix. This is important from a numerical point of view, since the non-convolutive structure disables the use of fast algorithms using e.g. Fast Fourier Transforms (FFTs) in the computations.

By collecting all data $\boldsymbol{y}_{i, j}$ columnwise in a single $N_{\mathrm{el}}^{2}$-point vector $\boldsymbol{y}$, we can now build the model:

$$
\boldsymbol{y}=\mathbf{H o}+\boldsymbol{n},
$$

where $\boldsymbol{o}$ is the $N_{x} N_{z}$-point column vector containing the discretized reflectivity map at each point of the reconstruction grid, $\mathbf{H}$ is the $N_{\mathrm{el}}^{2} N_{t} \times N_{x} N_{z}$ waveform matrix which is built by blocks corresponding to each emitter/receiver pair, and $\boldsymbol{n}$ is an uncertainty term representing model errors, measurement noise, etc. The waveform matrix $\mathbf{H}$ links temporal information in the data to spatial information in the unknown reflectivity map. Its construction is detailed in Figure 3. In practice, the waveform matrix $\mathbf{H}$ is too large to be stored in memory, for most configurations of realistic problems.

Let us remark that the TFM algorithm defined in Equation (1) is equivalent, up to discretization errors, to the linear relation:

$$
\boldsymbol{o}_{\mathrm{TFM}}=\mathbf{B}^{t} \boldsymbol{y}
$$

where $\mathbf{B}$ is a binary matrix with the same structure as the waveform matrix $\mathbf{H}$, where all waveforms are replaced by Kronecker deltas. Similarly, the Excitelet algorithm defined in Equation (3) can also be rewritten, after discretization, as the matrix-vector product:

$$
\boldsymbol{o}_{\mathrm{EXC}}=\mathbf{H}^{t} \boldsymbol{y}
$$

\section{B. Definition of the elementary signature}

The elementary signature represents the response of a potential scatterer located in the material in the received time-domain signal. It is mostly due to the electro-acoustical response of the transducers. In this paper, we make several 
simplifying assumptions that are discussed hereafter. First, the medium is supposed homogeneous and non-dispersive, so that the shape of the elementary signature is independent from the propagation distance. In the case of attenuative and dispersive media, the distortion of the elementary signature by frequencydependent attenuation could be considered [42]-[44]. Then, we also assume that the directivity patterns of the transducers do not depend on the frequency, and therefore only affect the amplitude of the elementary waveform (and not its shape). Apodization functions for far-field directivity [45], [46] are often considered in current beamforming techniques to account for these amplitude changes [4], [6] ${ }^{1}$. Here, we simply discard the data corresponding to emitter-receiver pairs that are too far from each other, and for which the directivity patterns may distort the corresponding response. From these two assumptions, the elementary signature can be supposed invariant with respect to the spatial location, that is, $\boldsymbol{h}_{i, j}^{i_{x}, i_{z}}=\boldsymbol{h}_{i, j}$ with the notations in Subsection III-A. Finally, we make the very common assumption that the elementary signature is similar for all elements. That is, $\boldsymbol{h}_{i, j}=\boldsymbol{h}, \forall i, j$.

In the following, we consider a Gaussian wavelet model for $\boldsymbol{h}$, which has been extensively used in the literature in order to model ultrasonic echoes [47], [48]. In particular, it assumes that the envelope of the response is symmetric. Some hints can be found in [49] to model echoes with more complex shapes. Following [47], [48], the Gaussian wavelet model can be written:

$$
h(t, \boldsymbol{\Theta})=e^{-\alpha t^{2}} \cos \left(2 \pi f_{o} t+\phi\right), \text { with } \Theta=\left[\alpha, f_{0}, \phi\right],
$$

where $f_{0}$ is the center frequency, $\phi$ is the phase shift and $\alpha$ is linked to the pulse width of the Gaussian function. Equivalently, the bandwidth ratio $\mathrm{BWR}_{p}$ at $p \mathrm{~dB}$ can be defined as:

$$
\mathrm{BWR}_{p}=\frac{\Delta f(p)}{f_{0}}
$$

where $\Delta f(p)$ is the width of the frequency band for a loss of $p \mathrm{~dB}$. Then, in the Gaussian case, $\alpha$ and $\mathrm{BWR}_{p}$ are linked by the following equation:

$$
\alpha=-\frac{\left(\pi \mathrm{BWR}_{p} f_{o}\right)^{2}}{4 \ln \left(10^{p} / 20\right)} .
$$

The center frequency and the bandwidth ratio can be approximately set knowing the transducer properties. Alternately, the waveform parameters can be estimated on targeted echoes in the data. Although the wavelet model (10) is not linear in $\Theta$, a nonlinear least-squares fitting procedure (e.g., based on the Levenberg-Marquardt algorithm) can be accurately initialized by the knowledge of the transducer properties (at least for $\alpha$ and $f_{0}$ ). Results obtained using generic parameters of the wavelet model and optimized ones will be compared in Subsection VI-D.

\section{INVERSION PROCEDURE}

In this section, we build an image reconstruction method from FMC data, based on the model in Equation (7).

${ }^{1} \mathrm{SB}:$ je ne fais pas le lien

\section{A. Naive inversion}

The naive estimation procedure in a least-squares (LS) sense computes the generalized inverse $\boldsymbol{o}_{\mathrm{LS}}[50]$ :

$$
\boldsymbol{o}_{\mathrm{LS}}=\arg \min _{\boldsymbol{o}}\|\boldsymbol{y}-\mathbf{H} \boldsymbol{o}\|^{2}=\left(\mathbf{H}^{T} \mathbf{H}\right)^{-1} \mathbf{H}^{T} \boldsymbol{y} .
$$

Acoustical signals are emitted and received in a limited frequency range close to the center frequency of the transducers. This means that available data do not contain all the information required for image reconstruction-in particular, highfrequency information containing details is mostly filtered out. Thus, the waveform matrix $\mathbf{H}$ is badly conditioned and the least-squares solution $\boldsymbol{o}_{\mathrm{LS}}$ is not satisfactory [51]. An example on experimental data will be shown in Subsection VI-B, where the condition number of matrix $\mathbf{H}^{t} \mathbf{H}$ is estimated at $10^{16}$.

\section{B. Inversion with a sparse-and-smooth prior}

In order to reconstruct information that is outside the bandwidth of the transducers, we adopt a regularization strategy where the solution is defined as the minimizer of the penalized least-squares criterion [51]:

$$
\widehat{\boldsymbol{o}}=\arg \min _{\boldsymbol{o}} J(\boldsymbol{o}), \text { with } J(\boldsymbol{o})=\|\boldsymbol{y}-\mathbf{H o}\|^{2}+\phi(\boldsymbol{o}) .
$$

The regularization function $\phi(\boldsymbol{o})$ is then designed in order to favor expected properties of the reconstructed image. For most NDT problems, materials under inspection may be considered homogeneous with only few scatterers [21], [22], that is, the reflectivity map is sparse. However, the size of the flaws may exceed the size of the pixels of the reconstruction grid, that is, scatterers may have some spatial extension in the reconstructed image. Therefore, we consider the two-term penalization function:

$$
\phi(\boldsymbol{o})=\mu_{1}\|\boldsymbol{o}\|_{1}+\mu_{2}\|\mathbf{D} \boldsymbol{o}\|^{2},
$$

where $\mathbf{D}$ is a matrix computing differences between values at neighbor pixels [52]. The $\ell_{1}$-norm penalization term is known to promote sparsity [53], whereas the second term balances sparsity with spatial smoothness. The cost function in (14) is convex, so that it can be minimized with local optimization strategies. However, the $\ell_{1}$-norm term is not differentiable at any vector containing zeros. We perform the optimization task by the FISTA algorithm (Fast Iterative Shrinkage Thresholding Algorithm) [54], which was shown to efficiently minimize such criteria. It relies on alternating between a gradient descent step on the differentiable part of the cost function and a thresholding step corresponding to the $\ell_{1}$-norm part. Its implementation then requires numerous evaluations of matrix-vector products involving $\mathbf{H}$ and $\mathbf{H}^{T}$. As explained in Subsection III-A, due to the inspection geometry, no simple structure in matrix $\mathbf{H}$ can be exploited for fast computations and, given the high dimensionality of the data, matrix $\mathbf{H}$ cannot be stored in memory. Our implementation relies on GPU in order to compute matrix-vector products. The elementary signature is stored and computations are parallelized on the fly for each pixel $\left(i_{x}, i_{z}\right)$.

Let us remark that a similar penalization framework was proposed in [55], where the waveform matrix $\mathbf{H}$ is replaced by 
the binary matrix B introduced at the end of Subsection III-A. This simplified version is numerically more efficient and achieves better resolution than the TFM thanks to the sparsityinducing penalization. However, by neglecting the elementary signature, it is not appropriate for separating overlapping echoes.

\section{Tuning of the regularization parameters}

Regularization parameters $\mu_{1}$ and $\mu_{2}$ balance between the least-squares fit and the desired properties of the solution. Their tuning can be done empirically or using calibration steps. Note that the parameter $\mu_{1}$ admits an upper bound, denoted $\mu_{1}^{\max }$, above which the reconstructed solution is identically zero. For the standard $\ell_{1}$-norm penalization case (that is, $\mu_{2}=0$ in (15)), we have [56]:

$$
\mu_{1}^{\max }=2\left\|\mathbf{H}^{t} \boldsymbol{y}\right\|_{\infty},
$$

where $\|\boldsymbol{x}\|_{\infty}$ denotes the maximum absolute value in vector $\boldsymbol{x}$. This bound is tight, which means that for $\mu_{1}<\mu_{1}^{\max }$, the solution is not identically zero. We prove in Appendix A that this bound is still valid for the two-term penalization (15), whatever the value of $\mu_{2}$. As a consequence, the two parameters can be tuned separately. In the following, we set $\mu_{1}$ to some fraction of $\mu_{1}^{\max }$, which controls the sparsity of the reconstructed image. Then, $\mu_{2}$ is set to a small positive value. In all our experiments, satisfactory solutions were obtained for a wide range of small positive values of parameter $\mu_{2}$.

\section{RESUlTS WITH SIMULATED DATA}

\section{A. Presentation of the synthetic model}

The goal of this section is to evaluate the capability of the proposed method to resolve close flaws. Synthetic data are generated using the model (7). The simulated waveform is the Gaussian wavelet defined in Equation (10), with center frequency $f_{0}=5 \mathrm{MHz}$ and $\mathrm{BWR}_{-6 \mathrm{~dB}}=40 \%$, corresponding to $\alpha=0.19$ and the phase is set to $\phi=0$. The sound velocity is $5000 \mathrm{~m} / \mathrm{s}$, such that the wavelength in the material is $\lambda=1 \mathrm{~mm}$. The pixel size in the simulated reflectivity map is $5 \mu \mathrm{m} \times 5 \mu \mathrm{m}$. Gaussian white noise with $10 \mathrm{~dB}$ SNR is added. The simulated reflectivity map is composed of two close point reflectors, whose spacing varies from $\lambda / 4$ to $\lambda$. The depth of the flaws is $z=20 \lambda$, and the flaws are located below the center of the ultrasonic probe, which contains 64 elements, with an inter-element distance of $\lambda / 2$.

Both the Excitelet algorithm and the inverse method are implemented using the "true" waveform. The pixel size used for image reconstruction is $20 \mu \mathrm{m} \times 20 \mu \mathrm{m}$, that is, four times bigger than the pixel size used for data generation. Consequently, the observation model (7) contains inaccuracies due to discretization. For these simulations, the regularization parameter $\mu_{2}$ is set to zero because the flaws have no spatial extension in the synthetic specimen, and $\mu_{1}=0.2 \mu_{1}^{\max }$.

\section{B. Metrics}

The difficulty to resolve two close flaws is evaluated with the Rayleigh criterion [18], [19], defined by

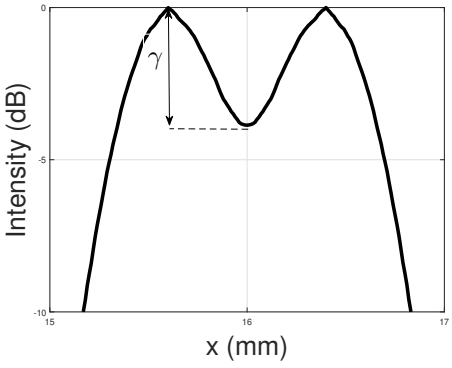

Fig. 4. Definition ot the peak to center intensity difference (PCID) criterion.

$\mathcal{R}=0.61 \lambda / \sin (\theta)$, where $\tan (\theta)=D /(2 z)$, with $D$ the aperture of the probe and $z$ the inspection depth. It defines the resolution limit of an imaging system [31]. In [19], the peak to center intensity difference (PCID) is introduced in order to evaluate the separation between two flaws. This criterion, denoted $\gamma$, is illustrated in Figure 4 and corresponds to the minimum value of intensity in the pixels that separate the two maxima corresponding to the flaws. In this paper, we consider that two point scatterers are not resolved if the PCID is above $-6 \mathrm{~dB}$. For the TFM and Excitelet images, which still contain oscillations, a post-processing step extracting the envelope in each image column is applied.

\section{Separation of close flaws}

Figure 5 shows the reconstructed images obtained by TFM, Excitelet and the proposed inverse method, and Figure 6 represents the horizontal profile of images at the flaw depth. The TFM and the Excitelet algorithm are able to resolve flaws which are separated by more than $\lambda$, which is in agreement with the Rayleigh criterion, equal to $0.97 \lambda$ in this case. The inverse method is able to resolve the two flaws in all cases. In particular, flaws distant of $\lambda / 4$ are well separated, which represents a resolving power four times superior to the Rayleigh limit.

Finally, simulations were performed by varying the distance between the flaws and for different bandwidth ratios (BWRs) of the waveform. Figure 7 compares the PCID for the different methods as a function of the distance between the two scatterers for different BWRs. For all methods, the resolving power increases with the BWR, which was expected since the pulse length decreases. The TFM and the Excitelet algorithm have approximately the same resolving power. In particular, the two scatterers appear as a single spot in the reconstructed image if they are separated by less than $0.65 \lambda$. The PCID of these two methods is lower than $-6 \mathrm{~dB}$ if their separation is greater than $0.9 \lambda$. On the contrary, the inverse method achieves a perfect PCID (equal to $-\infty \mathrm{dB}$ because sparsity enforces pixel intensities to zero between them) as soon as their distance is greater than $0.2 \lambda$.

\section{RESULTS WITH EXPERIMENTAL DATA}

\section{A. Presentation of the experiments}

In this section, our inversion method is tested on an aluminum block specimen. Experimental data are acquired using the 128-channel Pioneer platform from TPAC (West 


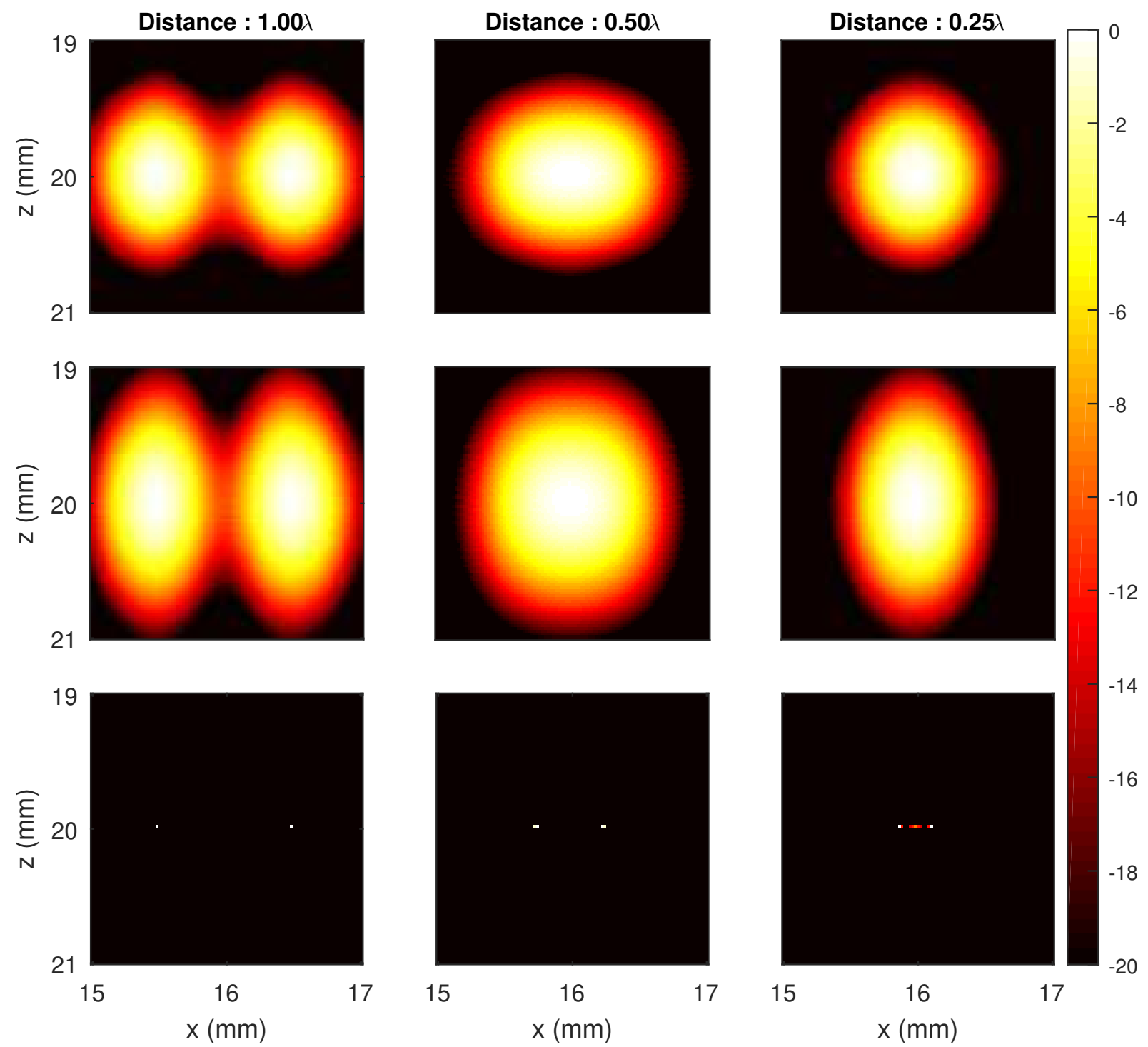

Fig. 5. Image reconstruction by TFM ( $1^{\text {st }}$ row), Excitelet ( $2^{\text {nd }}$ row) and our inverse method with $\mu_{1}=0.2 \mu_{1}^{\max }$ and $\mu_{2}=0$ ( $3^{\text {rd }}$ row) on a synthetic specimen containing two close flaws. Pixel values are in logarithmic scale.
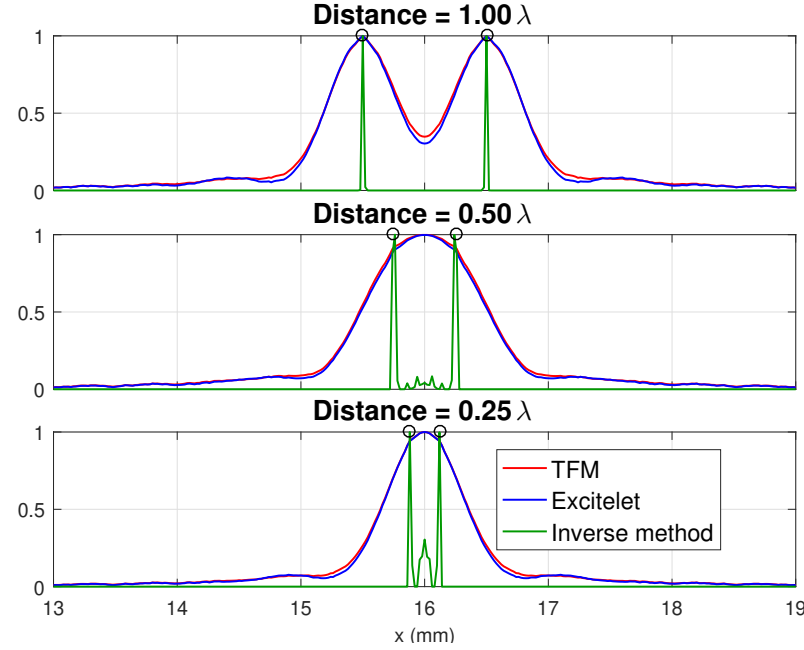

Fig. 6. Intensity of the TFM, Excitelet and inverse method reconstructions at the depth ( $z$ coordinate) of the two flaws $\left(\mu_{1}=0.2 \mu_{1}^{\max }\right.$ and $\left.\mu_{2}=0\right)$ Pixel intensities have been rescaled between 0 and 1 .

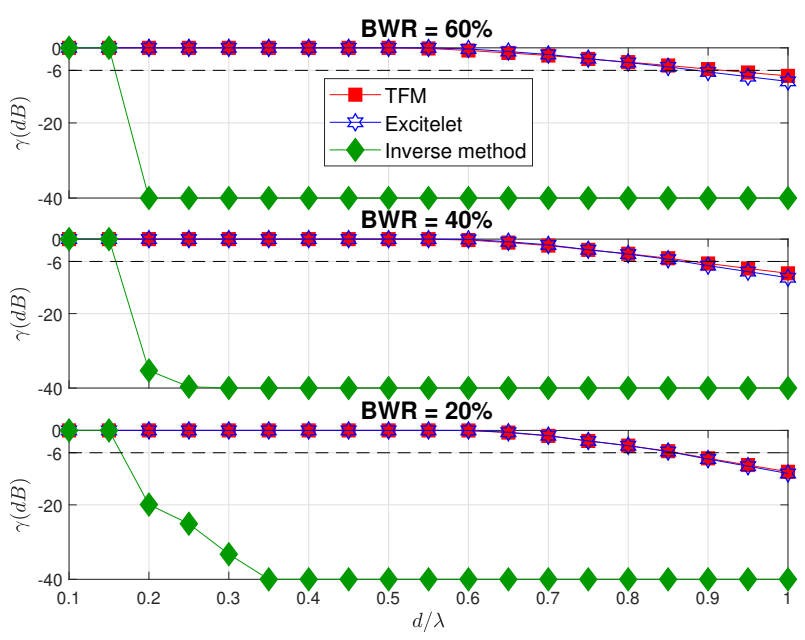

Fig. 7. Peak to center intensity difference for the three compared methods as a function of the distance between the two flaws $\left(\mu_{1}=0.2 \mu_{1}^{\max }\right.$ and $\left.\mu_{2}=0\right)$. 


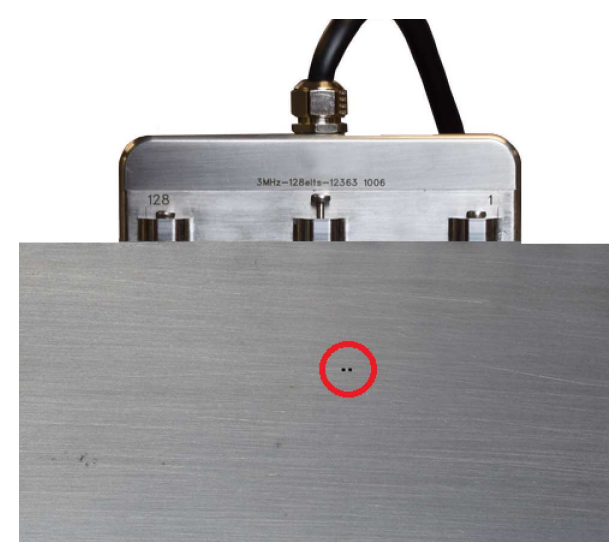

(a) Experiment \#1

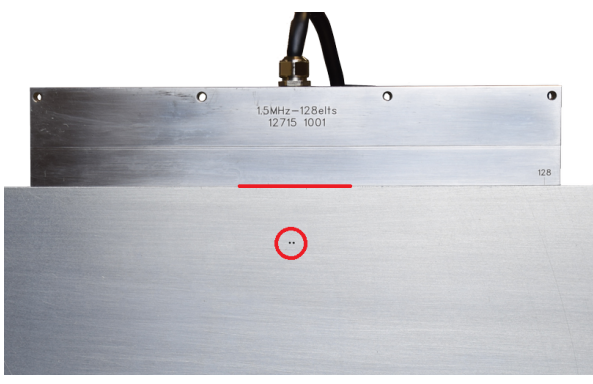

(b) Experiment \#2

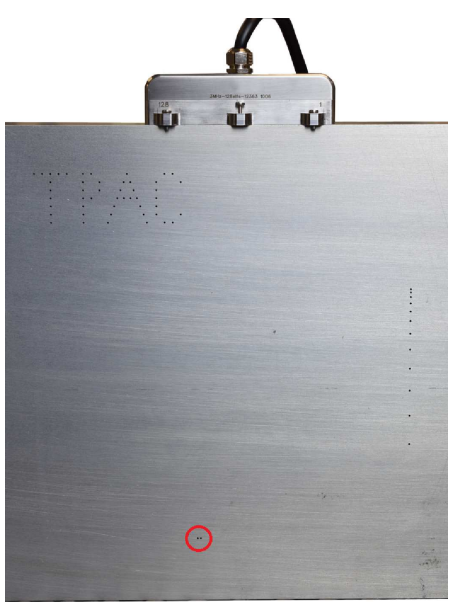

(c) Experiment \#3

Fig. 8. Inspected piece and corresponding probe for the three experiments in Section VI. The red circle locates the two side drilled holes.

TABLE I

PARAMETERS OF THE THREE EXPERIMENTS

\begin{tabular}{|l|c|c|c|c|c|c|}
\hline & $N_{\mathrm{el}}$ & $\begin{array}{c}f_{o} \\
(\mathrm{MHz})\end{array}$ & $\begin{array}{c}\lambda \\
(\mathrm{mm})\end{array}$ & $\begin{array}{c}\text { pitch } \\
(\mathrm{mm})\end{array}$ & $\begin{array}{c}\text { depth } \\
(\mathrm{mm})\end{array}$ & $\begin{array}{c}\mathcal{R} \\
(\mathrm{mm})\end{array}$ \\
\hline Exp \#1 & 96 & 3 & 2.10 & 0.8 & 40 & $1.86(\sim 0.89 \lambda)$ \\
\hline Exp \#2 & 32 & 1.5 & 4.20 & 2 & 40 & $4.18(\sim 1.00 \lambda)$ \\
\hline Exp \#3 & 128 & 3 & 2.10 & 0.8 & 260 & $6.68(\sim 3.18 \lambda)$ \\
\hline
\end{tabular}

TABLE II

PARAMETERS OF THE GAUSSIAN WAVELET USED IN THE EXPERIMENTS

\begin{tabular}{|c|c|c|c|}
\hline & $\begin{array}{c}f_{o} \\
(\mathrm{MHz})\end{array}$ & $\begin{array}{c}\text { BWR-6 } \\
(\%)\end{array}$ & $\begin{array}{c}\phi \\
(\mathrm{rad})\end{array}$ \\
\hline Exp \#1 & 3 & 30 & 0 \\
\hline Exp \#2 & 1.5 & 30 & 0 \\
\hline $\begin{array}{c}\text { Exp \#3 } \\
\text { (non-estimated) }\end{array}$ & 3 & 30 & 0 \\
\hline $\begin{array}{c}\text { Exp \#3 } \\
\text { (estimated) }\end{array}$ & 2.81 & 130 & 3.3 \\
\hline
\end{tabular}

Chester, Ohio, USA), and two probes from Imasonic (Voraysur-l'Ognon, France). The flaws are two 1-mm-diameter side drilled holes (SDH) with $1 \mathrm{~mm}$ edge-to-edge distance. Assuming that the maxima in the reflectivity map are located at the top of the SDH, the distance between the two corresponding maxima in the ultrasonic image should be $2 \mathrm{~mm}$. In all experiments, the specimen is inspected in contact and is supposed homogeneous, with the sound velocity equal to $6300 \mathrm{~m} / \mathrm{s}$.

Three experiments are presented, whose configurations are displayed in Figure 8. Their corresponding parameters are listed in Table I. The parameters of the Gaussian wavelets that used in the naive inversion, Excitelet and the inverse method are listed in Table II.

The inversion method is first compared with the TFM and the naive least-squares inversion. It aims to show the ill-posed nature of the problem, even in the favorable case where the two flaws are well separated in the TFM image. In the second experiment, a lower frequency is chosen and the inverse method is compared to the TFM and to the Excitelet method, in order to evaluate its ability to separate close flaws. The third experiment considers a mode difficult inspection configuration, where the test piece is inspected from the bottom side. The Rayleigh criterion is then the largest among the three experiments. In this last experiment, we study the influence of the waveform parameters that are used in the inverse method.

\section{B. Experiment \#1: Example of naive inversion}

In the first experiment, the piece is inspected using a 128-element probe pulsing at $3 \mathrm{MHz}$ with an inter-element distance (pitch) of $0.8 \mathrm{~mm}$. A picture of the inspection is shown in Figure 8 (a). Since the aperture of the probe is large (around $100 \mathrm{~mm}$ ), only the 96 central elements are used to acquire the data in order to discard signals for which the emitter-receiver distance is too large. The Rayleigh criterion is approximately $1.86 \mathrm{~mm} \sim 0.89 \lambda$, which is almost twice the edge-to-edge distance between the two flaws. The elementary signature is the Gaussian wavelet defined in Equation (10), with $f_{0}=3 \mathrm{MHz}, \mathrm{BWR}_{-6}=30 \%$ and $\phi=0$. Images obtained by the TFM, least-squares inversion and the proposed method are presented in Figure 9. The flaws are roughly resolved in the TFM image, with a PCID approximately equal to $-8 \mathrm{~dB}$. In the least-squares reconstruction, artifacts are too strong to identify any object in the image. This example shows that even for a low level of noise, the least-squares solution cannot provide satisfactory results. The inverse method clearly resolves the two flaws, as shown in Figure 9 (c). The distance between the two detected flaws is $1.92 \mathrm{~mm}$, which is a very accurate estimate.

C. Experiment \#2: Separation of close flaws with lowfrequency inspection

We now consider a lower frequency inspection of the former aluminum block, in order to create a more difficult 
(a)

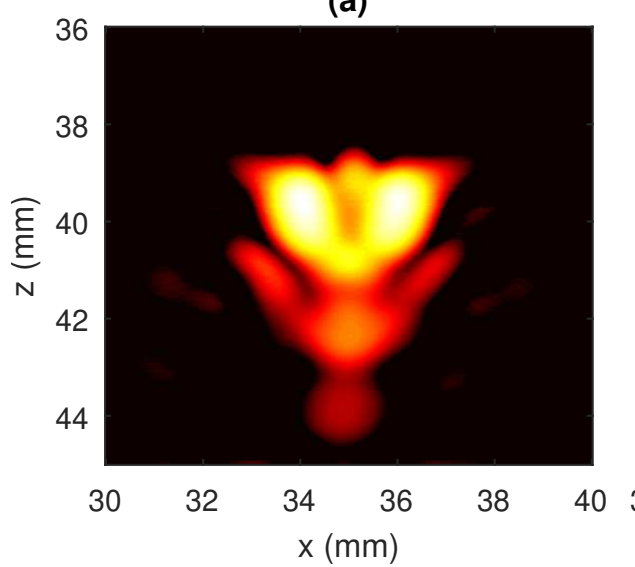

(b)

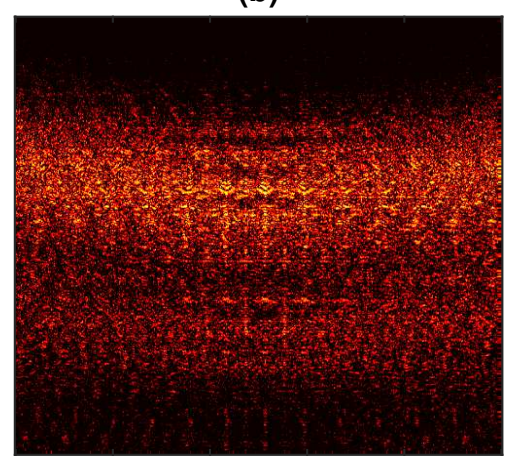

(c)

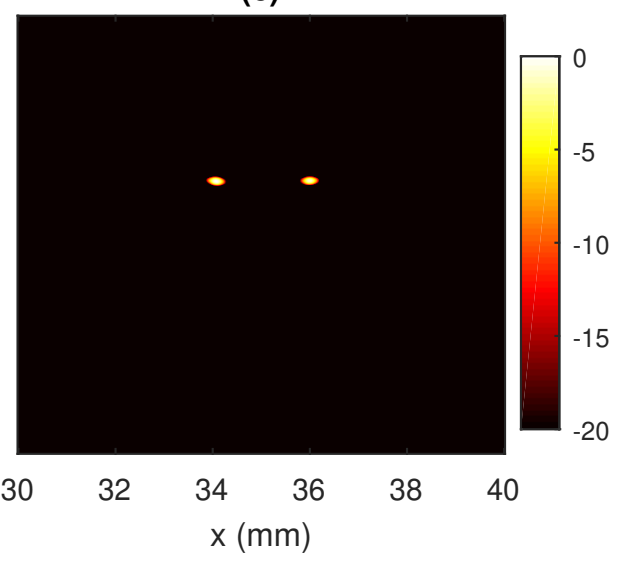

Fig. 9. Reconstructed images for experiment \#1. (a): TFM image, (b): least-squares inversion, and (c): reconstruction by the inverse method ( $\mu_{1}=0.6 \mu_{1}^{\text {max }}$ and $\mu_{2}=10^{-2}$ ). Pixel amplitudes are in logarithmic scale.

(a)

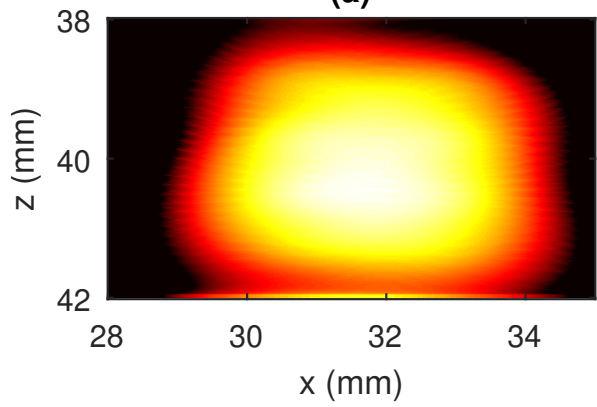

(b)

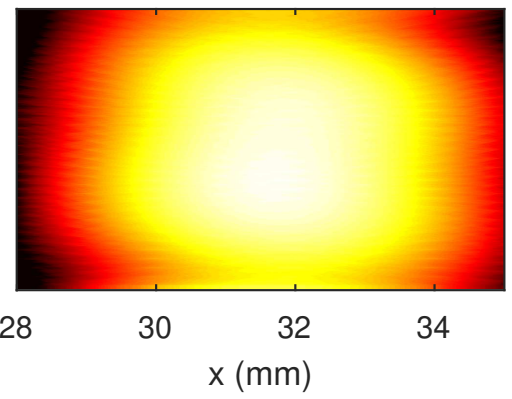

(c)

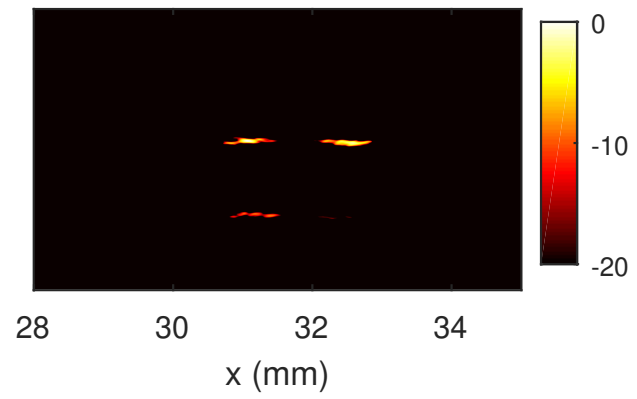

Fig. 10. Reconstructed images for experiment \#2. (a): TFM image, (b): Excitelet image, and (c): reconstruction by the inverse method ( $\mu_{1}=0.6 \mu_{1}^{\text {max }}$ and $\left.\mu_{2}=5 \cdot 10^{-3}\right)$. Pixel amplitudes are in logarithmic scale.

scenario. The probe frequency is now $1.5 \mathrm{MHz}$ and the pitch is $2 \mathrm{~mm}$. The wavelength is $\lambda=4.2 \mathrm{~mm}$, so that the edgeto-edge distance between the two flaws is approximately $\lambda / 4$. The configuration of the experiment is shown in Figure 8 (b). As in the first experiment, the aperture of the probe is very large (approximately $250 \mathrm{~mm}$ ), therefore only the 32 central elements of the probe are used to acquire the data. The resolution limit according to the Rayleigh criterion is $4.18 \mathrm{~mm}$, that is, of the order of $\lambda$. In this configuration, separating the flaws distant of $1 \mathrm{~mm}$ represents a resolving power four times superior to the Rayleigh criterion. The waveform used for both the Excitelet and the inverse method is the Gaussian wavelet in Equation (10) with $f_{0}=1.5 \mathrm{MHz}, \mathrm{BWR}_{-6}=30 \%$, and $\phi=0$. Results obtained by the TFM, Excitelet and the inverse method are shown in Figure 10. The two flaws appear like a single unresolved spot in both the TFM and the Excitelet images. On the contrary, the two spots are clearly separated in the image reconstructed with the inverse method. The maximum intensity values corresponding to these flaws are distant of $1.50 \mathrm{~mm}$, which is a bit less than the expected $2 \mathrm{~mm}$. This difference may be due to the large pitch of the probe used in this experiment, which impacts the lateral resolution.

\section{Experiment \#3: Influence of the elementary waveform}

Finally, we study the impact of the waveform parameters on the image quality. We consider a more difficult problem, where the two close flaws are located much farther from the probe. In the previous experiments, the parameters of the Gaussian echo model were set using generic values. In this experiment, we compare the results obtained by using either generic values or parameters that are estimated from the data.

The configuration of the experiment is shown in Figure 8 (c). The distance between the probe and the flaws is now $260 \mathrm{~mm}$. The same probe is used as in Subsection VI-B, with 128 elements, $3-\mathrm{MHz}$ center frequency and $0.8-\mathrm{mm}$ pitch. The distance between the two flaws is only $\lambda / 2$, and resolving the two flaws is very difficult because of the large inspection depth, which increases the Rayleigh criterion to $6.68 \mathrm{~mm} \sim 3.18 \lambda$.

As shown in Figure 11 (a), the two flaws cannot be separated in the TFM image. Our inversion procedure is first applied using generic waveform parameters, as in the first experiment $\left(f_{0}=3 \mathrm{MHz} \mathrm{BWR}_{-6}=30 \%\right.$ and $\left.\phi=0\right)$. The reconstructed image is shown in Figure 11 (b). It cannot clearly separate the two flaws, and it contains many artifacts that compensate the low adequacy between the data and the model. This image is typical of an inversion result with an inaccurate elementary waveform: side lobes are visible, whose intensity is similar to that of the main lobe. Last, our inversion procedure is 
(a)

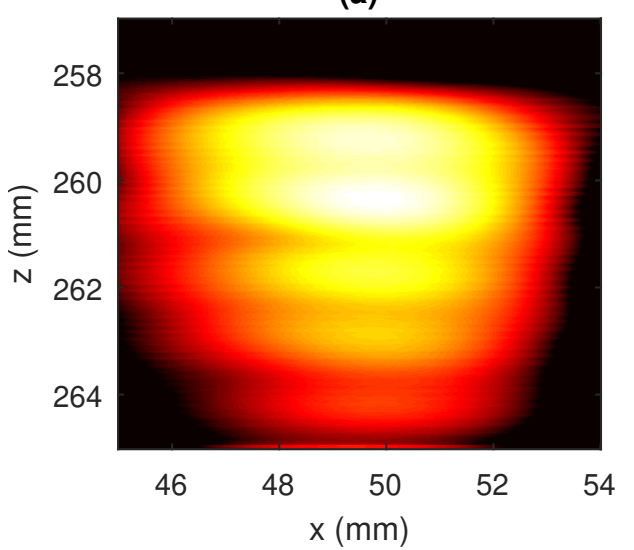

(b)

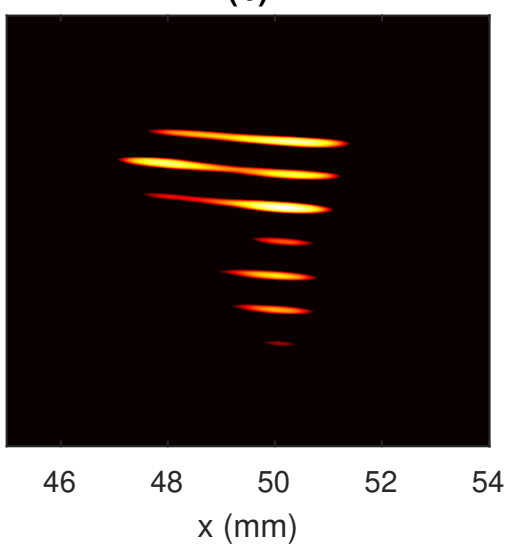

(c)

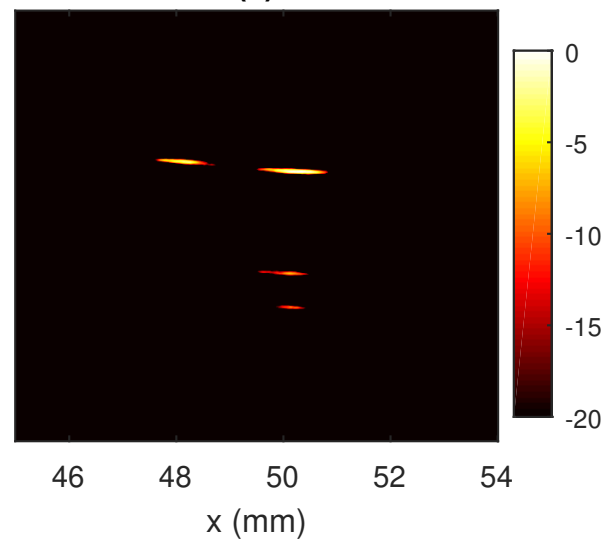

Fig. 11. Reconstructed images for experiment \#3. (a): TFM image, (b): inverse method using a generic waveform, (c): inverse method using an estimated waveform. For (b) and (c), $\mu_{1}=0.35 \mu_{1}^{\max }$ and $\mu_{2}=5.10^{-2}$. Pixel amplitudes are in logarithmic scale.

applied using waveform parameters that have been previously estimated from the data. More precisely, we consider the echoes reflected by the backside of the piece-located $40 \mathrm{~mm}$ deeper than the two flaws-for close emitter-receiver pairs. The Gaussian wavelet model (10) is then fitted to each timedomain signal with the Levenberg-Marquardt nonlinear least squares algorithm, and parameters are averaged among the estimates for which the lowest residual error was obtained. Estimated parameters are $f_{0}=2.81 \mathrm{MHz}, \mathrm{BWR}_{-6}=130 \%$, and $\phi=3.3 \mathrm{rad}$. Figure $11(\mathrm{c})$ shows she image obtained by our inversion procedure using these waveform parameters. The two flaws are now clearly separated, and the maxima of the two spots are distant of $1.97 \mathrm{~mm}$, which is very close to the 2-mm actual separation distance. This represents a resolving power six times above the Rayleigh criterion. Moreover, residual artifacts now have much lower intensity compared to the image in Figure 11 (b).

\section{CONCLUSION AND PERSPECTIVES}

In this paper, we have built a forward model which linearly relates the FMC data to the reflectivity map. The proposed imaging method consists in inverting this model to compute high-quality images from noisy datasets, by incorporating prior sparsity and spatial smoothness information on the reconstructed image. The ability of the method to resolve close scatterers has been demonstrated, with a resolution limit up to five times the Rayleigh criterion on synthetic data (up to six times on experimental data). On difficult problems where the scatterers are far from the inspection probe, we also showed that estimating specific parameters for the transducers response could significantly improve the image quality. From a methodological point of view, a joint approach aiming at simultaneously estimating the reflectivity image and the waveform parameters seems very attractive.

Our inversion methodology has been evaluated on an aluminum sample with a standard FMC acquisition procedure. A similar model could also be built for different kinds of data such as SAFT [10], PWI [11] or VSA [12]. Furthermore, the inverse method is not limited to contact inspection and could also be applied to more complex setups or specimens such as weld inspection using wedges [57], adaptive imaging [36], etc.

In the current implementation of the iterative optimization algorithm, the computation time can reach several minutes, depending on the complexity of the acquisition setup. However, we noted that, in practice, rather satisfactory images compared to TFM could be obtained after only a few iterations, that is, a few seconds of computation time. Therefore, a faster approach with a limited number of iterations could also be competitive. In order to reduce the computation time, another inversion approach would formulate an inverse problem starting from the TFM image, by considering the TFM image as a backprojection of the data in the spatial domain. A linear inverse problem could then be formulated in the image domain with a much smaller number of data points, at the expense of some loss of information [58], [59].

Last, in this paper, the method has been tested on a simple material, for which the elementary signature was considered as shift invariant. For dispersive materials, attenuation and dispersion could be integrated in the procedure, which would modify the shape of the waveform as a function of the propagation distance [42]-[44]. By predicting the waveform distortion with appropriate models, such a method may be a promising solution in order to reconstruct high-quality ultrasonic images of scattering materials.

\section{APPENDIX A}

UPPER BOUND ON PARAMETER $\mu_{1}$ IN THE PENALIZATION FUNCTION (15)

We prove that the minimizer of the cost function $J$ defined by Equations (14)-(15) is identically zero if and only if $\mu_{1} \geq \mu_{1}^{\max }$, with $\mu_{1}^{\max }=2\left\|\mathbf{H}^{t} \boldsymbol{y}\right\|_{\infty}$. This result is already known in the standard $\ell_{1}$-norm penalization case $\left(\mu_{2}=0\right)$, see for example [56]. In our case, we can rewrite the quadratic part of $J$ as:

$$
\|\boldsymbol{y}-\mathbf{H} \boldsymbol{o}\|^{2}+\mu_{2}\|\mathbf{D} \boldsymbol{o}\|^{2}=\left\|\boldsymbol{y}_{e}-\mathbf{H}_{e} \boldsymbol{o}\right\|^{2},
$$




$$
\begin{aligned}
\text { where } \mathbf{H}_{e}= & {\left[\begin{array}{c}
\mathbf{H} \\
\sqrt{\mu_{2}} \mathbf{D}
\end{array}\right] \text { and } \boldsymbol{y}_{e}=\left[\begin{array}{l}
\boldsymbol{y} \\
0
\end{array}\right], \text { so that: } } \\
& J(\boldsymbol{o})=\left\|\boldsymbol{y}_{e}-\mathbf{H}_{e} \boldsymbol{o}\right\|^{2}+\mu_{1}\|\boldsymbol{o}\|_{1} .
\end{aligned}
$$

Now, applying the result in [56], we have that the minimizer of (18) is identically zero if and only if $\mu_{1} \geq \mu_{1}^{\max }$, with:

$$
\mu_{1}^{\max }=2\left\|\mathbf{H}_{e}^{t} \boldsymbol{y}_{e}\right\|_{\infty}=2\left\|\mathbf{H}^{t} \boldsymbol{y}\right\|_{\infty} .
$$

\section{ACKNOWLEDGMENT}

This work was partially funded by the French ANRT (Association Nationale Recherche Technologie), project 2017/1083.

\section{REFERENCES}

[1] J. Krautkramer and H. Krautkramer, Ultrasonic Testing of materials. Berlin: Springer-Verlag, 1990.

[2] M. Fatemi and A. C. Kak, "Ultrasonic B-scan imaging: Theory of image formation and a technique for restoration," Ultrasonic Imaging, vol. 2, no. 1, pp. 1-47, January 1980.

[3] C. R. Farrar and K. Worden, "An introduction to structural health monitoring," Philosophical Transactions of the Royal Society A: Mathematical, Physical and Engineering Sciences, vol. 365, no. 1851, pp. 303-315, Feb. 2007.

[4] B. Drinkwater and P. Wilcox, "Ultrasonic arrays for non-destructive evaluation: A review," NDT\&E INT, vol. 39, no. 7, pp. 525-541, Oct. 2006.

[5] P. Wilcox, C. Holmes, and B. Drinkwater, "Advanced Reflector Characterization with Ultrasonic Phased Arrays in NDE Applications," IEEE Trans. Ultrason., Ferroelectr., Freq. Control, vol. 54, no. 8, pp. 15411550, Aug. 2007

[6] C. Holmes, B. Drinkwater, and P. Wilcox, "Post-processing of the full matrix of ultrasonic transmit-receive array data for non-destructive evaluation," NDT\&E INT, vol. 38, no. 8, pp. 701-711, Dec. 2005.

[7] A. Caulder, "Full Matrix Capture and Total Focusing Method: The Next Evolution in Ultrasonic Testing," Materials Evaluation, p. 7, 2018.

[8] M. Tanter and M. Fink, "Ultrafast imaging in biomedical ultrasound," IEEE Trans. Ultrason., Ferroelectr., Freq. Control, vol. 61, no. 1, pp. 102-119, Jan. 2014

[9] M. Karaman, P.-C. Li, and M. O. Donnell, "Synthetic aperture imaging for small scale systems," IEEE Trans. Ultrason., Ferroelectr., Freq. Control, vol. 42, no. 3, pp. 429-442, May 1995.

[10] J. A. Seydel, "Ultrasonic synthetic-aperture focusing techniques in NDT," Research techniques in nondestructive testing, pp. Vol. 6, pp. $1-47,1982$.

[11] G. Montaldo, M. Tanter, J. Bercoff, N. Benech, and M. Fink, "Coherent plane-wave compounding for very high frame rate ultrasonography and transient elastography," IEEE Trans. Ultrason., Ferroelectr., Freq. Control, vol. 56, no. 3, pp. 489-506, Mar. 2009.

[12] M.-H. Bae and M.-K. Jeong, "A study of synthetic-aperture imaging with virtual source elements in B-mode ultrasound imaging systems," IEEE Trans. Ultrason., Ferroelectr, Freq. Control, vol. 47, no. 6, pp. 1510-1519, Nov. 2000.

[13] M. Sutcliffe, P. Charlton, and M. Weston, "Multiple virtual source aperture imaging for non-destructive testing," Insight - Non-Destructive Testing and Condition Monitoring, vol. 56, no. 2, pp. 75-81, Feb. 2014.

[14] R.-H. Stolt, "Migration by Fourier Transform," Geophysics, vol. 43, no. 1, pp. 23-48, Feb. 1978.

[15] D. Garcia, L. L. Tarnec, S. Muth, E. Montagnon, J. Poree, and G. Cloutier, "Stolt's f-k migration for plane wave ultrasound imaging," IEEE Trans. Ultrason., Ferroelectr, Freq. Control, vol. 60, no. 9, pp. 1853-1867, Sep. 2013.

[16] A. Hunter, B. Drinkwater, and P. Wilcox, "The wavenumber algorithm for full-matrix imaging using an ultrasonic array," IEEE Trans. Ultrason., Ferroelectr., Freq. Control, vol. 55, no. 11, pp. 2450-2462, Nov. 2008.

[17] T. Stepinski, "An Implementation of Synthetic Aperture Focusing Technique in Frequency Domain," IEEE Trans. Ultrason., Ferroelectr., Freq. Control, vol. 54, no. 7, pp. 1399-1408, Jul. 2007.

[18] J. Rayleigh, "XXXI. Investigations in optics, with special reference to the spectroscope," The London, Edinburgh, and Dublin Philosophical Magazine and Journal of Science, vol. 8, no. 49, pp. 261-274, Oct. 1879.
[19] C. Fan, M. Caleap, M. Pan, and B. W. Drinkwater, "A comparison between ultrasonic array beamforming and super resolution imaging algorithms for non-destructive evaluation," Ultrasonics, vol. 54, no. 7, pp. 1842-1850, Sep. 2014

[20] N. Quaegebeur and P. Masson, "Correlation-based imaging technique using ultrasonic transmit-receive array for Non-Destructive Evaluation," Ultrasonics, vol. 52, no. 8, pp. 1056-1064, Dec. 2012.

[21] A. Tuysuzoglu, J. M. Kracht, R. Cleveland, M. Çetin, and W. Karl, "Sparsity driven ultrasound imaging," J. Acoust. Soc. Am., vol. 131, no. 2, pp. 1271-1281, Feb. 2012.

[22] G. Guarneri, D. Pipa, F. Junior, L. de Arruda, and M. Zibetti, "A Sparse Reconstruction Algorithm for Ultrasonic Images in Nondestructive Testing," Sensors, vol. 15, no. 4, pp. 9324-9343, Apr. 2015.

[23] T. Szasz, A. Basarab, and D. Kouame, "Beamforming Through Regularized Inverse Problems in Ultrasound Medical Imaging," IEEE Trans. Ultrason., Ferroelectr., Freq. Control, vol. 63, no. 12, pp. 2031-2044, Dec. 2016.

[24] E. Ozkan, V. Vishnevsky, and O. Goksel, "Inverse Problem of Ultrasound Beamforming With Sparsity Constraints and Regularization," IEEE Trans. Ultrason., Ferroelectr., Freq. Control, vol. 65, no. 3, pp. 356365, Mar. 2018.

[25] S.-K. Sin and C.-H. Chen, "A comparison of deconvolution techniques for the ultrasonic nondestructive evaluation of materials," IEEE Trans. Image Process., vol. 1, no. 1, pp. 3-10, Jan. 1992.

[26] E. Carcreff, S. Bourguignon, J. Idier, and L. Simon, "Resolution enhancement of ultrasonic signals by up-sampled sparse deconvolution," in 2013 IEEE International Conference on Acoustics, Speech and Signal Processing, May 2013, pp. 6511-6515.

[27] A. Besson, D. Perdios, F. Martinez, M. Arditi, Y. Wiauxy, and J. Thiran, "Ussr: An ultrasound sparse regularization framework," in 2017 IEEE Int. Ultrason. Symp., Sep. 2017.

[28] A. Besson, D. Perdios, F. Martinez, Z. Chen, R. E. Carrillo, M. Arditi, Y. Wiaux, and J. Thiran, "Ultrafast ultrasound imaging as an inverse problem: Matrix-free sparse image reconstruction," IEEE Trans. Ultrason., Ferroelectr, Freq. Control, vol. 65, no. 3, pp. 339-355, 2018.

[29] E. Carcreff, N. Laroche, Z. Xu, and D. Braconnier, "A quantitative study of tfm-like imaging techniques for nondestructive evaluation," in 12 th European Conference on Non-destructive Testing, 2018.

[30] F. Simonetti, "Multiple scattering: The key to unravel the subwavelength world from the far-field pattern of a scattered wave," Phys. Rev. E, vol. 73, no. 3, Mar. 2006.

[31] — - "Localization of pointlike scatterers in solids with subwavelength resolution," Applied Physics Letters, vol. 89, no. 9, pp. 094-105, Aug. 2006.

[32] C. Fan, M. Pan, F. Luo, and B. Drinkwater, "Multi-frequency timereversal-based imaging for ultrasonic nondestructive evaluation using full matrix capture," IEEE Trans. Ultrason., Ferroelectr., Freq. Control, vol. 61, no. 12, pp. 2067-2074, Dec. 2014.

[33] Y. Labyed and L. Huang, "Ultrasound time-reversal MUSIC imaging with diffraction and attenuation compensation," IEEE Trans. Ultrason., Ferroelectr., Freq. Control, vol. 59, no. 10, Oct. 2012.

[34] M. Sutcliffe, M. Weston, B. Dutton, P. Charlton, and K. Donne, "Realtime full matrix capture for ultrasonic non-destructive testing with acceleration of post-processing through graphic hardware," NDT\&E INT, vol. 51 , pp. $16-23,2012$.

[35] G. D. Connolly, M. J. S. Lowe, J. A. G. Temple, and S. I. Rokhlin, "The application of Fermat's principle for imaging anisotropic and inhomogeneous media with application to austenitic steel weld inspection," Proceedings of the Royal Society A: Mathematical, Physical and Engineering Sciences, vol. 465, no. 2111, pp. 3401-3423, Nov. 2009.

[36] L. Le Jeune, S. Robert, P. Dumas, A. Membre, and C. Prada, "Adaptive ultrasonic imaging with the total focusing method for inspection of complex components immersed in water," AIP Conference Proceedings, vol. 1650 , pp. 1037-1046, 2015.

[37] M. Weston, P. Mudge, C. Davis, and A. Peyton, "Time efficient autofocussing algorithms for ultrasonic inspection of dual-layered media using Full Matrix Capture," NDT\&E INT, vol. 47, pp. 43-50, Apr. 2012.

[38] T. J. Moser, "Shortest path calculation of seismic rays," Geophysics, vol. 56, no. 1, pp. 59-67, Jan. 1991.

[39] J. A. Sethian, "A fast marching level set method for monotonically advancing fronts." Proceedings of the National Academy of Sciences, vol. 93, no. 4, pp. 1591-1595, Feb. 1996.

[40] A. J. Brath and F. Simonetti, "Phased Array Imaging of ComplexGeometry Composite Components," IEEE Trans. Ultrason., Ferroelectr., Freq. Control, vol. 64, no. 10, pp. 1573-1582, Oct. 2017.

[41] N. Laroche, E. Carcreff, S. Bourguignon, J. Idier, and A. Duclos, "An inverse approach for ultrasonic imaging by total focusing point for close reflectors separation," in 2018 IEEE Int. Ultrason. Symp., Oct 2018. 
[42] K. V. Gurumurthy and R. Martin Arthur, "A dispersive model for the propagation of ultrasound in soft tissue." Ultrasonic Imaging, vol. 4, pp. 355-377, 1982.

[43] R. Kuc, "Modeling acoustic attenuation of soft tissue with a minimumphase filter,' Ultrasonic Imaging, vol. 6, no. 1, pp. 24 - 36, 1984.

[44] E. Carcreff, S. Bourguignon, J. Idier, and L. Simon, "A linear model approach for ultrasonic inverse problems with attenuation and dispersion," IEEE Trans. Ultrason., Ferroelectr., Freq. Control, vol. 61, no. 7, pp. 1191-1203, Jul. 2014

[45] A. R. Selfridge, G. S. Kino, and B. T. Khuri-Yakub, "A theory for the radiation pattern of a narrow-strip acoustic transducer," Applied Physics Letters, vol. 37, no. 1, pp. 35-36, Jul. 1980.

[46] A. C. Clay, S.-C. Wooh, L. Azar, and J.-Y. Wang, "Experimental Study of Phased Array Beam Steering Characteristics," p. 13, 1999.

[47] R. Demirli and J. Saniie, "Model based time-frequency estimation of ultrasonic echoes for NDE applications," in 2000 IEEE Int. Ultrason. Symp., vol. 1, Oct. 2000, pp. 785-788.

[48] R. Demirli and J. Saniie, "Model-based estimation of ultrasonic echoes. Part I: Analysis and algorithms," IEEE Trans. Ultrason., Ferroelectr., Freq. Control, vol. 48, no. 3, pp. 787-802, May 2001.

[49] - "Asymmetric Gaussian chirplet model and parameter estimation for generalized echo representation," Journal of the Franklin Institute, vol. 351, no. 2, pp. 907-921, Feb. 2014.

[50] G. Golub and C. Van Loan, Matrix Computations (3rd Ed.). Baltimore, MD, USA: Johns Hopkins University Press, 1996.
[51] J. Idier, Bayesian Approach to Inverse Problems. London, U.K.: ISTE Ltd and John Wiley \& Sons Inc, Apr. 2008.

[52] B. Hunt, "The inverse problem of radiography," Mathematical Biosciences, vol. 8, pp. 161-179, Jun. 1970.

[53] M. O'Brien, A. Sinclair, and S. Kramer, "Recovery of a sparse spike time series by 11 norm deconvolution," IEEE Trans. Signal Processing, vol. 42, pp. 3353 - 3365, 011995.

[54] A. Beck and M. Teboulle, "A Fast Iterative Shrinkage-Thresholding Algorithm for Linear Inverse Problems," SIAM Journal on Imaging Sciences, vol. 2, no. 1, pp. 183-202, 2009.

[55] E. Carcreff, N. Laroche, D. Braconnier, A. Duclos, and S. Bourguignon, "Improvement of the total focusing method using an inverse problem approach," in 2017 IEEE Int. Ultrason. Symp., Sep. 2017.

[56] J.-J. Fuchs, "More on sparse representations in arbitrary bases," IFAC Proceedings Volumes, vol. 36, no. 16, pp. 1315 - 1320, 2003.

[57] J. Zhang, B. W. Drinkwater, P. D. Wilcox, and A. J. Hunter, "Defect detection using ultrasonic arrays: The multi-mode total focusing method," NDT \& E International, vol. 43, no. 2, pp. 123-133, Mar. 2010.

[58] A. Besson, L. Roquette, D. Perdios, M. Simeoni, M. Arditi, P. Hurley, Y. Wiaux, and J.-P. Thiran, "A Physical Model of Non-stationary Blur in Ultrasound Imaging," IEEE Trans. Comput. Imaging, pp. 1-1, 2019.

[59] N. Laroche, S. Bourguignon, E. Carcreff, J. Idier, and A. Duclos, "Fast inverse approach for the deconvolution of ultrasonic TFM images using a spatially varying PSF in NDT," in 2019 IEEE Int. Ultrason. Symp., Oct 2019. 